\title{
Tendencias del suicidio en Colombia, 1985-2002
}

\author{
Ricardo Cendales, ${ }^{1}$ Claudia Vanegas, ${ }^{1}$ Marco Fierro, ${ }^{1}$ \\ Rodrigo Córdoba y Ana Olarte ${ }^{1}$
}

Forma de citar Cendales R, Vanegas C, Fierro M, Córdoba R, Olarte A. Tendencias del suicidio en Colombia, 1985-2002. Rev Panam Salud Publica. 2007;22(4):231-8.

RESUMEN Objetivos. Describir las tendencias de la mortalidad por suicidios en Colombia en el período de 1985 a 2002, analizar su comportamiento según el sexo, el grupo de edad y el mecanismo de lesión y calcular los años de vida potencial perdidos (AVPP) por suicidios en ese período.

Métodos. Se calcularon las tasas de mortalidad crudas, específicas por edad y sexo y ajustadas por la edad, a partir de la información de mortalidad y población de las bases de datos oficiales del Departamento Administrativo Nacional de Estadística de Colombia. Se estimaron los AVPP, corregidos por el impacto social, la edad y la mala calidad de los registros de muertes. La tabulación de los resultados se realizó según los códigos X600-X849 y Y870 de la décima revisión de la Clasificación Estadística Internacional de Enfermedades y Problemas Relacionados con la Salud (CIE-10) y los códigos E950-E959 de la novena revisión (CIE-9). Resultados. Se observó un aumento considerable en las tasas de suicidios en Colombia a partir de 1998, particularmente en la población de adultos jóvenes y en hombres. Las tasas más altas en los hombres se observaron en los grupos de 20 a 29 años y de mayores de 70, con un incremento en el tiempo. En las mujeres, las tasas más altas se registraron en el grupo de 10 a 19 años. La proporción de AVPP por suicidios aumentó de 0,81\% en 1981 a 2,20\% en 2002. En los hombres, los principales mecanismos de lesión fueron las heridas por armas de fuego y explosivos, el ahorcamiento y el envenenamiento, con un aumento relativo del ahorcamiento, mientras que en las mujeres fue el envenenamiento.

Conclusiones. Se confirmó la tendencia ascendente en la tasa de suicidios en Colombia, especialmente en el segmento de la población laboralmente activa, lo que conlleva un drástico incremento en los AVPP.

Palabras clave Suicidio, años potenciales de vida perdidos, Colombia.

El suicidio constituye un problema de salud pública a escala mundial. En

\footnotetext{
1 Centro de Investigaciones del Sistema Nervioso, Departamento de Investigaciones, Cundinamarca, Colombia. La correspondencia se debe dirigir a Ricardo Cendales, CISNE-Investigaciones, Carrera 69 No. 170-40, Bogotá, Colombia. Correo electrónico: acardocen@yahoo.com
}

un estudio sobre la carga de enfermedades, realizado por la Organización Mundial de la Salud (OMS) en 1990, el suicidio, junto con otras lesiones autoinfligidas, ocupó la quinta posición por su importancia en el segmento de la población entre 15 y 44 años de edad. Por esta causa se perdieron en total 14645000 años de vida saludable en el año 1990 (1) y se cree que el número de víctimas anuales podría ascender a cerca de un millón y medio antes del año 2020 (2). La OMS ha hecho un llamado de atención sobre el incremento de este problema a escala mundial. 
En los países industrializados, el suicidio es una de las principales causas de muerte prematura y aunque las tasas de mortalidad por suicidios han disminuido en la población mayor de 50 años, han aumentado en la población de adultos jóvenes (3-7). Esta situación incrementa sustancialmente el número de años de vida potencial perdidos (AVPP). En términos simples, 40 AVPP pueden ser el resultado de la muerte de una persona de 30 años de edad que tenía una esperanza de vida de 70 años o el resultado de la muerte de 10 personas de 70 años de edad que tenían una esperanza de vida de 74 años; por esta razón las muertes que ocurren más tempranamente representan un mayor número de AVPP y este indicador puede crecer aunque haya disminuido la tasa de mortalidad general por suicidios en la población. Este es el caso, por ejemplo, del Reino Unido, donde se observó un incremento en el número de AVPP a pesar de que la tasa de mortalidad por suicidios disminuyó, debido al aumento en el número de suicidios en los adultos jóvenes (4).

Las tasas de suicidios se han incrementado claramente entre 1950 y 2000. Según datos de la OMS de septiembre de 2007 (2), Lituania, Belarús y Rusia tienen las mayores tasas de suicidios en Europa, con cifras superiores a 60 por 100000 habitantes en hombres y a 10 por 100000 habitantes en mujeres, mientras que Kazajstán y Sri Lanka encabezan la lista de países de Asia con tasas superiores a 44,0 por 100000 habitantes en hombres y a 8,9 por 100000 habitantes en mujeres. Las tasas más bajas se presentan en los países musulmanes y en la Región de las Américas. Las mayores tasas en nuestra Región la tienen Guyana (42,5 por 100000 habitantes en hombres y 12,1 por 100000 habitantes en mujeres), seguida por Uruguay, Cuba, Canadá, Estados Unidos, Chile y Surinam, con tasas que varían entre 17,8 y 24,5 por 100000 habitantes en hombres y entre 3,1 y 6,6 por 100000 habitantes en mujeres (2). En los Estados Unidos de América, la tasa cruda en 2002 fue de 11,0 por 100000 habitantes $\mathrm{y}$, en contraste con lo que ocurre en el Reino Unido, las tasas mostraron un descenso en la población menor de 35 años, mientras que en el grupo de 35 a 54 años se evidenció un aumento entre 1993 y 2002 (8).

En este artículo se describen las tendencias de la mortalidad por suicidios en Colombia en el período de 1985 a 2002, se analiza su comportamiento según el sexo, el grupo de edad y el mecanismo de lesión y se calculan los AVPP por suicidios en ese período.

\section{MATERIALES Y MÉTODOS}

Los datos de mortalidad y de población se tomaron de las bases de datos oficiales del Departamento Administrativo Nacional de Estadística (DANE) de Colombia correspondientes al período de 1981 a 2002, aunque el análisis se centró fundamentalmente en el período 1985-2002. Se calcularon las tasas de mortalidad crudas, específicas por edad y sexo, y ajustadas por la edad mediante el método directo con la población mundial como referencia $(9,10)$. Dado que la estimación de las tasas de mortalidad se realizó a partir del análisis de todo el universo estudiado, no se emplearon intervalos de confianza ni pruebas de hipótesis. Se calcularon los AVPP por suicidios según el método de Murray $(11,12)$ y se empleó la esperanza de vida en Japón como referencia ideal. Como existe cierta incertidumbre acerca de cómo se habrían vivido los años restantes si la persona no hubiera muerto, se aplicó un factor de corrección que descontó 3\% al valor de cada AVPP a partir del primero, de manera que cada AVPP adicional tuvo un valor menor. Además, se utilizó una función exponencial para dar un valor diferencial a cada AVPP de acuerdo con el momento de la vida en el que ocurrió la muerte, lo que introdujo en el análisis el impacto social que causa la muerte prematura. Este abordaje se emplea para el análisis de los años de vida saludables perdidos y refleja de manera más fidedigna el impacto de la mortalidad prematura.

Para el cálculo de los AVPP se hicieron correcciones para ajustar la mala calidad de los registros de mortalidad: las muertes que no tenían información de edad se distribuyeron de forma proporcional entre las que tenían esa información; las muertes naturales certificadas por personal no médico se distribuyeron proporcionalmente entre las muertes naturales que habían sido certificadas por médicos; y las muertes con signos, síntomas o afecciones mal definidas se distribuyeron también proporcionalmente entre las muertes naturales. Los primeros dos ajustes se hicieron de manera específica por año, sexo y causa de muerte; el tercer ajuste se hizo por año y sexo.

Para el análisis de la mortalidad y de los AVPP no se hicieron correcciones del subregistro de la mortalidad, ya que en Colombia no se cuenta con cifras actualizadas. Estudios previos han demostrado que las cifras disponibles sobreestiman el subregistro y en consecuencia inflan de manera desmedida las cifras de mortalidad (13).

La agrupación de causas de mortalidad se realizó a partir de la información contenida en las listas de la Organización Panamericana de la Salud 6/61 (14) y 6/67 (15), esta última modificada para poder presentar las muertes violentas por suicidio separadas de las muertes por otras causas externas. Para la tabulación de los resultados se establecieron equivalencias entre la novena y décima revisiones de la Clasificación Estadística Internacional de Enfermedades y Problemas Relacionados con la Salud (CIE). Se emplearon los códigos X600-X849 y Y870 de la décima revisión (CIE-10) y los códigos E950-E959 de la novena revisión (CIE-9) para definir las muertes causadas por suicidio.

\section{RESULTADOS}

Con excepción de las afecciones mal definidas y las enfermedades infecciosas, todas las demás causas de muerte mostraron una tendencia al incremento en los últimos años y esta fue más marcada en el grupo de muertes por causas violentas (figura 1). El análisis específico de la mortalidad por suicidios reveló un aumento importante en sus tasas a partir del año 1998, 
FIGURA 1. Muertes por grandes grupos de causas, ${ }^{\mathrm{a}}$ Colombia, $1981-2002$

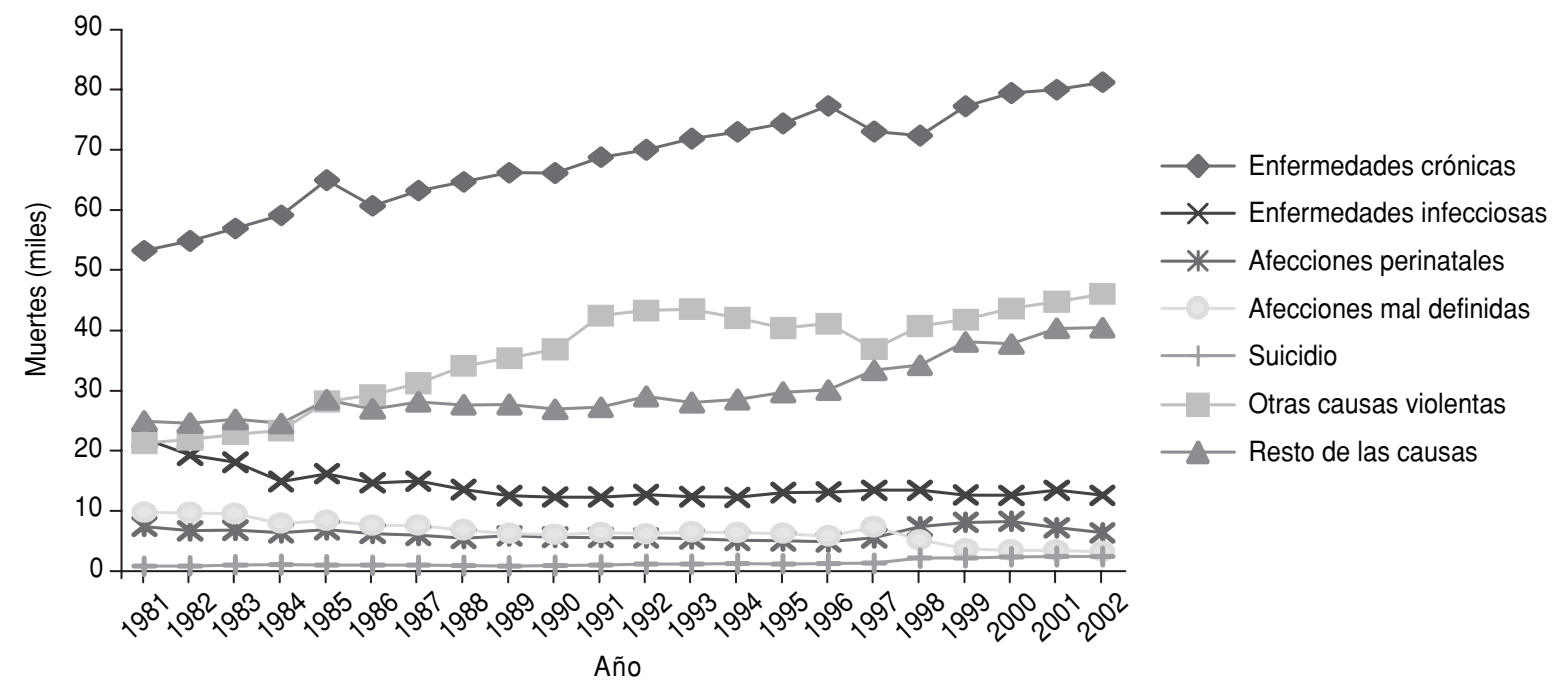

Fuente: Elaborada a partir de los datos del Departamento Administrativo Nacional de Estadística (DANE) de Colombia. a Según la lista modificada 6/67 de la Organización Panamerícana de la Salud (15).

después de un período estable entre los años 1981 y 1997; este aumento fue más notable en hombres que en mujeres (figura 2).

\section{Carga de la enfermedad por suicidios en Colombia}

En el año 2002 se contabilizaron 70482 AVPP por suicidios en Colombia. La proporción de AVPP por suicidios aumentó de $0,81 \%$ en 1981 a $2,20 \%$ en 2002 (figura 3). La mayor proporción de los AVPP se debió a muertes por otras causas violentas (cerca de $40 \%$ del total); esta cifra está estrechamente relacionada con el elevado número de homicidios y accidentes en la población joven en Colombia. Las muertes por enfermedades crónicas constituyen la segunda causa más importante de AVPP (cerca de 24\%).

\section{Comportamiento según el sexo y el grupo de edad}

Las tendencias en las tasas de mortalidad por suicidios en hombres mostraron tasas específicas más elevadas en los grupos de 20 a 29 años y de más de 70 años, con un incremento en el tiempo. Se debe destacar el incremento observado en el grupo de 20 a 29 años por tratarse de un segmento económicamente activo de la población (cuadro 1).

En las mujeres, los grupos de edad con mayores tasas específicas fueron el de 10 a 19 y el de 20 a 29 años, con un mayor incremento en el primero de esos grupos en el tiempo. Hasta 1997, el grupo de 20 a 29 años tenía las tasas específicas más altas, pero a partir de 1998 las mayores tasas correspondieron al grupo de 10 a 19 años. En contraste con los hombres, el grupo de mujeres mayores de 70 años tuvo tasas

FIGURA 2. Tasas de suicidios ajustadas por edad, por 100000 habitantes, Colombia, 1985-2002

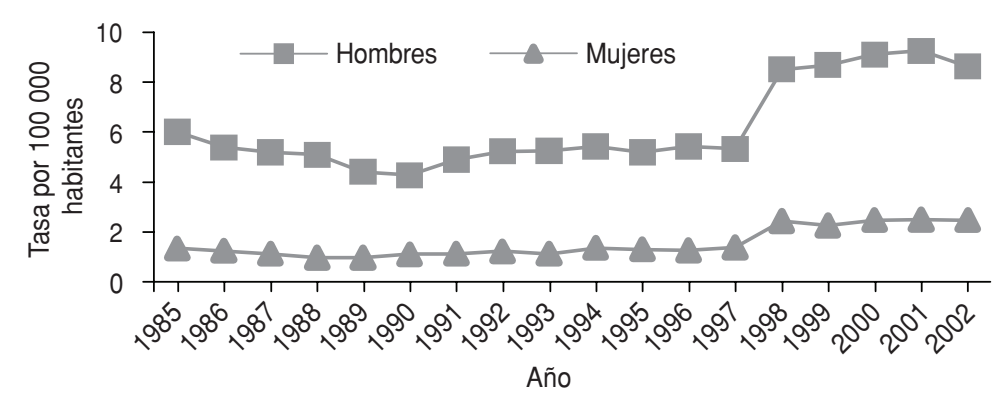

Fuente: Elaborada a partir de los datos del Departamento Administrativo Nacional de Estadística (DANE) de Colombia. 
FIGURA 3. Porcentaje de años de vida potencial perdidos (AVPP), según grandes grupos de causas de mortalidad, ${ }^{a}$ Colombia, 1981-2002

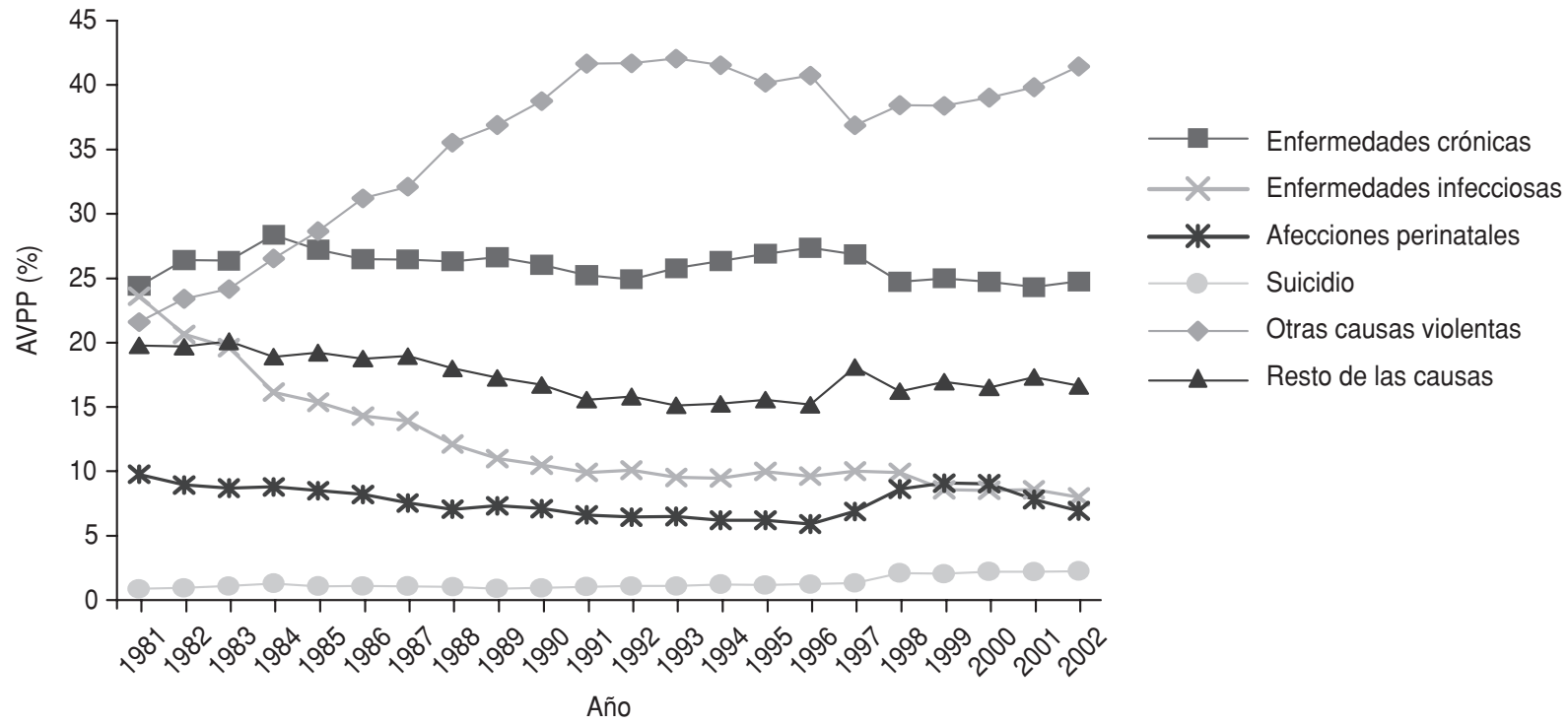

Fuente: Elaborada a partir de los datos del Departamento Administrativo Nacional de Estadística (DANE) de Colombia. a Causas agrupadas según la lista modificada 6/67 de la Organización Panamerícana de la Salud (15).

bajas y no mostró un incremento en el tiempo (cuadro 2).

La razón entre el número de suicidios en hombres y mujeres fue cercana a 1 para el grupo de edad de 10 a 19 años. Esta razón se incrementó gradualmente en los grupos de mayor edad y alcanzó sus valores máximos en las personas de 60 años o más. Sin embargo, la disminución en el tiempo de la razón entre el número de suicidios en hombres y mujeres en todos los grupos de edad en los últimos años indica un aumento relativo de los suicidios en las mujeres, lo cual se confirmó mediante el análisis de las tasas específicas por edad (cuadro 3).

\section{Mecanismos de lesión}

En los hombres, los tres principales mecanismos de lesión fueron las heridas por armas de fuego y explosivos, el ahorcamiento y el envenenamiento. En todos estos casos se observó un in- cremento en el tiempo, especialmente en el uso del ahorcamiento (figura 4).

En las mujeres, el mecanismo de lesión más frecuente fue el envenenamiento, con una clara tendencia al incremento a partir de 1998 (figura 5).

\section{DISCUSIÓN}

Tres hipótesis podrían explicar el aumento en las tasas de suicidios: un mejor registro de la mortalidad, un ar-

CUADRO 1. Tasas específicas de mortalidad por suicidios en hombres según el grupo de edad, por 100000 habitantes, Colombia, 1985-2002

\begin{tabular}{|c|c|c|c|c|c|c|c|c|c|c|c|c|c|c|c|c|c|c|}
\hline $\begin{array}{l}\text { Grupo de } \\
\text { edad } \\
\text { (años) }\end{array}$ & \multicolumn{18}{|c|}{ Año } \\
\hline 10 a 19 & 3,0 & 2,6 & 3,3 & 2,5 & 2,0 & 2,4 & 3,0 & 3,0 & 3,2 & 3,4 & 2,8 & 3,5 & 4,0 & 6,0 & 5,8 & 6,7 & 6,1 & 6,3 \\
\hline 20 a 29 & 9,9 & 9,6 & 10,4 & 9,4 & 7,5 & 7,5 & 9,3 & 9,6 & 9,2 & 9,7 & 9,4 & 8,6 & 9,6 & 15,7 & 15,3 & 18,1 & 17,4 & 17,1 \\
\hline 30 a 39 & 6,6 & 6,7 & 5,6 & 6,1 & 6,0 & 5,3 & 6,5 & 6,8 & 7,0 & 7,1 & 5,7 & 6,5 & 6,1 & 10,6 & 10,7 & 10,7 & 10,5 & 10,7 \\
\hline 40 a 49 & 8,0 & 7,8 & 5,6 & 6,1 & 5,9 & 5,5 & 5,4 & 5,7 & 6,0 & 6,4 & 6,6 & 6,9 & 5,6 & 8,4 & 9,0 & 9,8 & 10,6 & 10,0 \\
\hline 70 a 79 & 12,8 & 5,7 & 5,8 & 9,7 & 10,6 & 10,8 & 9,2 & 12,4 & 10,9 & 11,6 & 7,2 & 11,4 & 11,7 & 15,5 & 15,1 & 17,1 & 17,5 & 15,2 \\
\hline 80 o más & 12,1 & 7,6 & 11,2 & 16,5 & 11,2 & 7,4 & 5,1 & 3,8 & 8,3 & 14,8 & 13,3 & 11,3 & 14,8 & 21,3 & 21,8 & 22,2 & 15,3 & 16,0 \\
\hline
\end{tabular}

Fuente: Elaborado a partir de las bases de datos del Departamento Administrativo Nacional de Estadística (DANE) de Colombia. 
CUADRO 2. Tasas específicas de mortalidad por suicidios en mujeres según el grupo de edad, por 100000 habitantes, Colombia, 1985-2002

\begin{tabular}{|c|c|c|c|c|c|c|c|c|c|c|c|c|c|c|c|c|c|c|}
\hline $\begin{array}{l}\text { Grupo de } \\
\text { edad } \\
\text { (años) }\end{array}$ & \multicolumn{18}{|c|}{ Año } \\
\hline 10 a 19 & 2,1 & 2,3 & 2,0 & 1,4 & 1,5 & 1,6 & 1,7 & 2,1 & 1,7 & 2,2 & 1,9 & 2,5 & 2,1 & 5,5 & 4,9 & 5,5 & 5,6 & 6,2 \\
\hline 20 a 29 & 2,7 & 2,8 & 2,5 & 2,0 & 1,9 & 2,1 & 2,3 & 2,6 & 2,1 & 2,6 & 2,5 & 2,0 & 2,7 & 4,4 & 4,0 & 4,4 & 4,8 & 4,0 \\
\hline 30 a 39 & 1,3 & 1,0 & 0,8 & 1,5 & 1,4 & 1,4 & 1,4 & 1,3 & 1,3 & 1,6 & 1,6 & 1,6 & 1,6 & 2,0 & 2,2 & 2,1 & 2,2 & 2,0 \\
\hline 40 a 49 & 1,4 & 1,0 & 0,8 & 0,7 & 1,0 & 1,1 & 0,9 & 1,2 & 1,1 & 1,1 & 1,6 & 0,9 & 1,1 & 2,0 & 2,0 & 2,2 & 1,1 & 1,8 \\
\hline 70 a 79 & 0,6 & 1,2 & 0,6 & 0,9 & 0,8 & 1,1 & 0,5 & 0,3 & 0,7 & 1,5 & 0,7 & 1,1 & 1,3 & 0,4 & 1,2 & 1,4 & 1,3 & 0,7 \\
\hline 80 o más & 1,8 & 0 & 1,8 & 2,4 & 1,5 & 1,4 & 0,7 & 0 & 0 & 0,6 & 0,6 & 0,5 & 0 & 1,0 & 0,5 & 0,9 & 0,5 & 1,3 \\
\hline
\end{tabular}

Fuente: Elaborado a partir de las bases de datos del Departamento Administrativo Nacional de Estadística (DANE) de Colombia.

tificio técnico creado por cambios en el sistema de codificación de la mortalidad o un incremento real en el número de suicidios. La primera hipótesis no parece ser válida, ya que es evidente que el mayor incremento en el número de muertes de este período fue en las clasificadas como causas violentas. En cuanto a la segunda hipótesis, aunque el incremento en las tasas de suicido coincidió con el cambio del sistema de codificación de la mortalidad para el registro de estadísticas vitales en Colombia en 1997 (de la CIE-9 a la CIE-10), las codificaciones son equivalentes y en consecuencia no deben generar alteraciones en los registros. Se favorece, entonces, la hipótesis que indica un incremento real en la tendencia de los suicidios en Colombia.
El aumento descrito en las tasas de mortalidad por suicidios en Colombia en años recientes contrasta con las tasas estables encontradas en un estudio previo realizado entre 1973 y 1996 (16). Sin embargo, al analizar el comportamiento de las tasas durante períodos más largos se observa con mayor claridad la tendencia ascendente, con un aumento en la tasa ajustada por edad de 1,3 por 100000 habitantes en 1950 a 5,5 por 100 000 habitantes en 2002. Vale la pena mencionar que el subregistro de la mortalidad en Colombia dificulta la interpretación de las tendencias. Como se desconoce el porcentaje de subregistro, no es posible ajustar las tasas de suicidios; el último estudio sobre el subregistro de la mortalidad en Colombia se realizó en 1993. Sin embargo, el registro de la mortalidad ha mejorado en los últi- mos años con la implementación de la CIE-10 en 1997 y la revisión y perfeccionamiento de los procedimientos del sistema de registro de estadísticas vitales (datos no publicados).

En términos de carga de la enfermedad, el suicidio representó $2,20 \%$ de los AVPP en Colombia en 2002. El incremento en las tasas de suicido, especialmente en los grupos más jóvenes, lo explica. Este aumento es más notable en las mujeres de 10 a 19 años y en los hombres de 20 a 29 años. Un comportamiento similar se ha observado en algunos países europeos, como Irlanda, España y las repúblicas que formaban parte de la Unión Soviética, así como en Australia y Nueva Zelanda en Oceanía $(17,18)$.

Cuando se comparan las tasas de suicidios en el grupo de 10 a 19 años de

CUADRO 3. Razón entre el número de muertes por suicidio en hombres y en mujeres, según el grupo de edad, Colombia 1985-2002

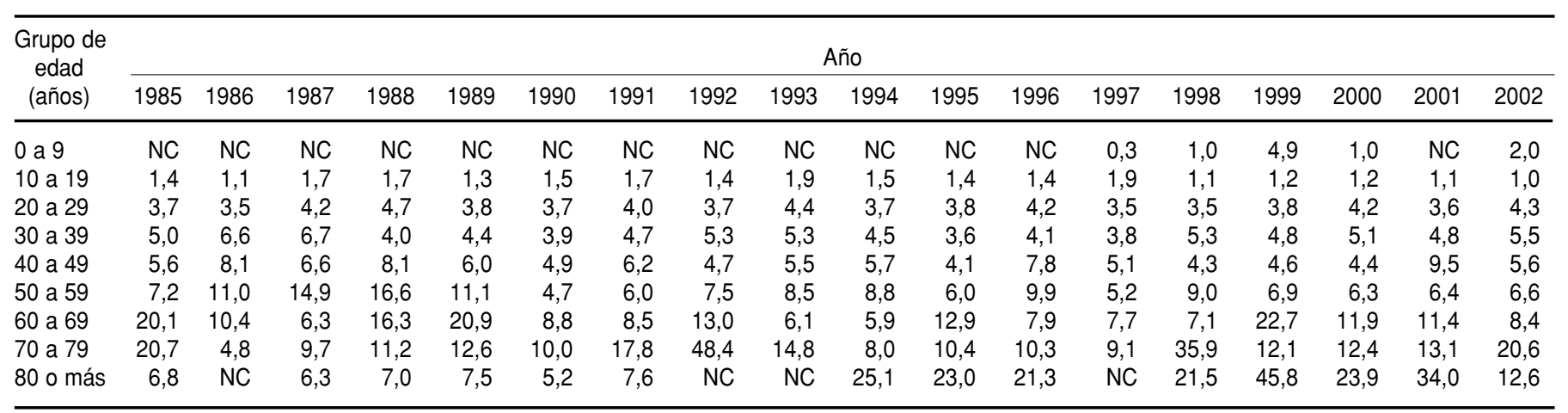

Fuente: Elaborado a partir de los datos del Departamento Administrativo Nacional de Estadística (DANE) de Colombia.

$\mathrm{NC}$ : Estas razones no se pudieron calcular por no haber muertes registradas en mujeres. 
FIGURA 4. Tasas específicas de mortalidad por suicidios en hombres, según el mecanismo de lesión. Colombia, 1985-2002

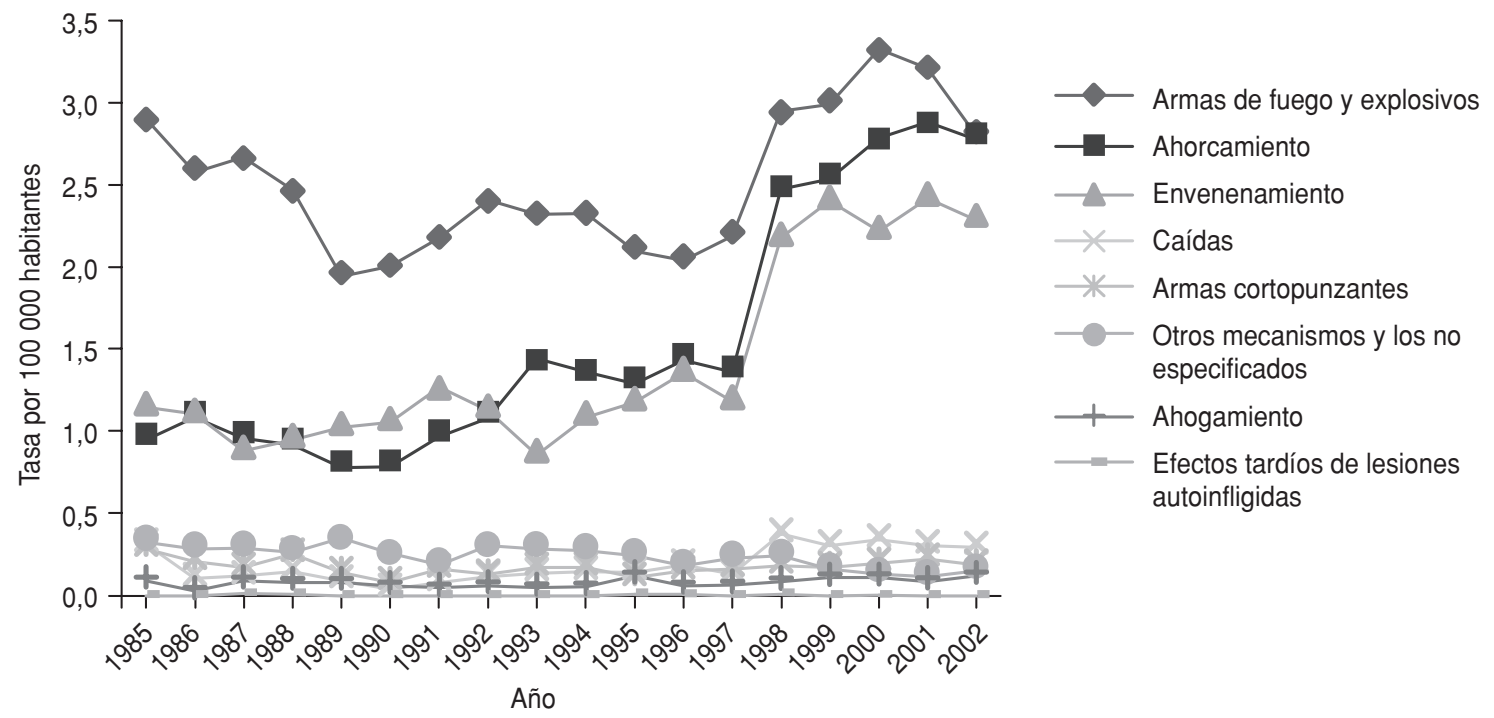

Fuente: Elaborada a partir de los datos del Departamento Administrativo Nacional de Estadística (DANE) de Colombia.

uno y otro sexos observadas en Colombia con las descritas para otros 47 países miembros de la OMS, se comprueba que la tasa observada en el grupo de mujeres colombianas es una de las más altas, mientras que en los hombres la tasa se mantiene en un nivel intermedio (18). Los únicos paí- ses que muestran una razón cercana a uno entre el número de suicidios en hombres y en mujeres en este grupo de edad son Cuba y China, mientras que

FIGURA 5. Tasas específicas de mortalidad por suicidios en mujeres, según el mecanismo de lesión, Colombia, 1985-2002

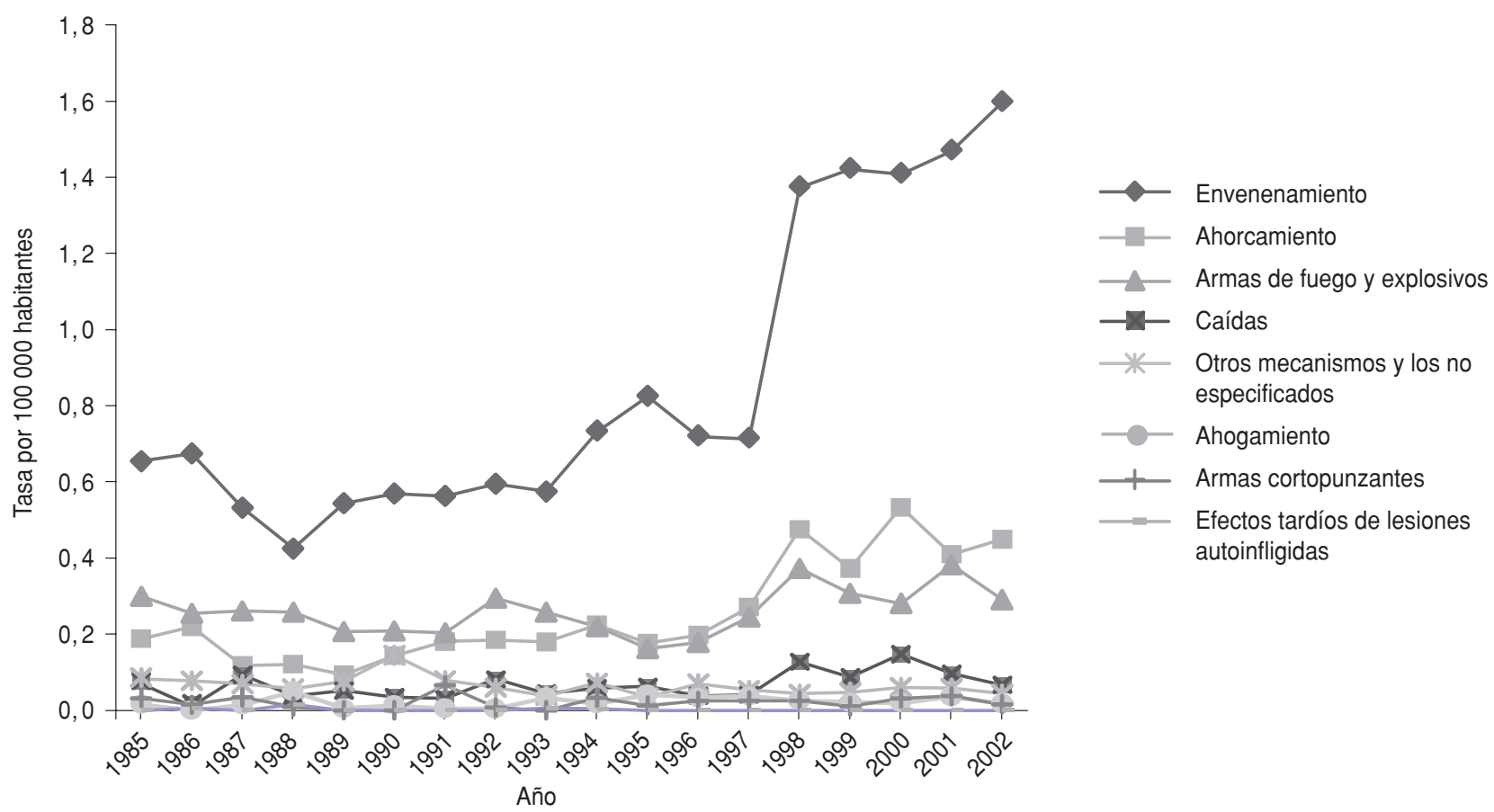

Fuente: Elaborada a partir de los datos del Departamento Administrativo Nacional de Estadística (DANE) de Colombia. 
la mayoría de los demás países tienen una razón cercana a 4:1 entre hombres y mujeres $(18,19)$. Otro estudio basado en los datos de cerca de 90 países llegó a conclusiones similares y señaló a China, Cuba, Ecuador, El Salvador y Sri Lanka como las excepciones (20).

La elevada razón entre el número de suicidios en hombres y en mujeres (cercana a 20:1) encontrada en la población mayor de 70 años fue superior a la informada en otros países. Este comportamiento parece deberse a la baja tasa de suicidios en las mujeres de este grupo de edad en Colombia.

Este estudio tiene algunas limitaciones. En primer lugar, la falta de corrección por el subregistro de la mortalidad puede alterar el resultado del análisis de las tendencias. Una segunda limitación está relacionada con el hecho de que en Colombia existen dos fuentes diferentes de información sobre las muertes por causas externas: el DANE, empleado en la presente investigación, y el Instituto Nacional de Medicina Legal y Ciencias Forenses (INML). ${ }^{2}$ Estas fuentes están desarticuladas y como consecuencia puede haber un subregistro en la mortalidad por suicidios.

Los resultados de este trabajo confirman la tendencia ascendente en la tasa de suicidios en Colombia, especialmente en el segmento de la población

2 Cada institución lleva registros independientes y, aunque todas las muertes deben estar registradas en ambas instituciones, esto no siempre ocurre, ya que un considerable número de muertes registradas por el DANE no pasan por el INML. El número de suicidios registrados por el INML en 2001 fue de 2056 (21) y en el DANE de 2 456; el INML informó 2045 suicidios en 2002 (22), mientras que el DANE registró 2423. laboralmente activa, lo que conlleva un drástico incremento en los AVPP.

$\mathrm{El}$ análisis de tendencias exige una corrección adecuada de la cifras de subregistro, particularmente en países como Colombia, donde se ha informado de una mejoría notable en la cobertura de las defunciones. Por ello se deben realizar estudios actualizados de las cifras de cobertura de la mortalidad. Se deben también estudiar las causas de la tendencia ascendente en la mortalidad por suicidios en la población de hombres y mujeres jóvenes en Colombia, particularmente en mujeres de 10 a 19 años. Se recomienda hacer un esfuerzo especial para articular las dos fuentes de información de mortalidad del país, lo que permitirá generar datos más confiables en estudios futuros.

\section{REFERENCIAS}

1. Bobes García J. La psiquiatría en esquemas. Prevención de comportamientos suicidas en esquemas. Barcelona: ARS Médica; 2005.

2. World Health Organization. Suicide rates per 100.000 by country, year and sex [sitio en internet]. Geneva: WHO; 2007. Hallado en http: / / www.who.int/mental_health / prevention/suicide_rates/en/index.html. Acceso el 25 de septiembre de 2007.

3. Sánchez R. Estudio de la imitación como factor de riesgo para ideación suicida en estudiantes universitarios adolescentes. Rev Colomb Psiquiatr. 2005;34(1):12-25.

4. Gunnell D, Middleton N. National suicide rates as an indicator of the effect of suicide on premature mortality. Lancet. 2003;362(9388); 961-2.

5. Centers for Disease Control and Prevention. Suicide among black youths. United States, 1980-1995. Morb Mort Wkly Rep. 1998;47(10) 193-6.

6. Yunes J, Rajs D. Tendencia de la mortalidad por causas violentas en la población general y entre los adolescentes y jóvenes de la Región de las Américas. Cad Saude Publica. 1994;10(1 suppl):1-47.

7. Bearman P, Moody J. Suicide and friendships among American adolescents. Am J Public Health. 2004;94(1):89-95.

8. American Association of Suicidology. USA suicide: 2004 oficial final data [sitio en Internet]. Washington, D.C.: American Association of Suicidology; 2006. Hallado en http:/ / www.suicidology.org/associations/1045/fil es / 2004OfficialFinalData.pdf. Acceso el 25 de septiembre de 2007. de Colombia; 2005.
9. World Health Organization. Age standardization of rates: a new WHO standard. Geneva: WHO. Hallado en http://www.emro.who. int/ncd/publications/WHO_pop_standard. pdf. Acceso el 25 de septiembre de 2007. (GPE Discusion Paper Series No. 31)

10. Santos Silva I. Medidas de la aparición de enfermedad y de otros acontecimientos relacionados con la salud. En: Santos Silva I. Epidemiología del cáncer: principios y métodos. Lyon: International Agency for Research in Cancer; 1999. Pp. 61-88.

11. Murray CJL, Rethinking DALYs. En: Murray CJL, López A. The global burden of disease. Cambridge: Harvard University Press; 1996.

12. Rodríguez J, Gallardo HM. Mortalidad y años de vida ajustados por discapacidad como medidas de la carga de la enfermedad, Colombia 1985-1995. Bogotá: Ministerio de Salud; 1999.

13. Cendales R. Estimación de los casos nuevos de cáncer en Colombia en el año 2005 [tesis de especialidad]. Bogotá: Universidad Nacional

14. Organización Panamericana de la Salud. Clasificación Internacional de Enfermedades: preparación de listas cortas para la tabulación de datos. Bol Epidemiol. 2002;23(4):3-6.

15. Organización Panamericana de la Salud. Nueva lista OPS 6/67 para la tabulación de datos de mortalidad CIE-10. Bol Epidemiol. 1999;20(3):4-9.

16. Gómez-Restrepo C, Rodríguez N, de Romero L, Pinilla C, López E, Díaz-Granados N, et al. Suicidio y lesiones autoinfligidas, Colombia, 1973-1996. Rev Colomb Psiquiatr. 2002;31(2): 123-36.
17. León M, Muñoz C, Pertusa C, Romero S. Tendencias de la mortalidad por suicidio en el norte y sur de Europa en la década de los noventa. Madrid: Universidad Autónoma de Madrid; 2007. Hallado en http://www. uam.es/departamentos/medicina/preven tiva/especifica/jornadas 2004/61_trabajosui cidio.htm. Acceso el 25 de septiembre de 2007.

18. Levi F, La Vecchia C, Lucchini F, Negri E, Saxena S, Maulik PK, et al. Trends in mortality from suicide, 1965-99. Acta Psichiatr Scand. 2003:108(5):341-9.

19. American Psychiatric Association. Practice guideline for the assessment and treatment of patients with suicidal behaviors. Am J Psychiatry. 2003;160(11 suppl):1-60.

20. Wasserman D, Cheng Q, Jiang GX. Global suicide rates among young people aged 15-19. World Psychiatry. 2005:4(2):114-20.

21. González J, Merchán C, Marthe A, López C, Ricaurte A, Restrepo A, et al. Suicidios en Colombia 2001. En: Instituto Nacional de Medicina Legal y Ciencias Forenses. Forensis 2001. Bogotá: Imprenta Nacional; 2001. Pp. 167-91.

22. Hernández W. Los suicidios en Colombia. Entre el libre albedrío y la prevención. En: Instituto Nacional de Medicina Legal y Ciencias Forenses, Fondo de Prevención Vial. Forensis 2002. Bogotá: Instituto Nacional de Medicina Legal y Ciencias Forenses; 2002. Pp. 111-27.

Manuscrito recibido el 24 de julio de 2006. Aceptado para publicación, tras revisión, el 21 de julio de 2007. 
ABSTRACT Objectives. To report trends in mortality from suicide in Colombia from 1985 to 2002 by sex, age group, and method, and determine the number of Years of Potential Life Lost (YPLL) to suicide during this period.

Suicide trends in Colombia, 1985-2002

Key words Suicide, mortality, Colombia. poison was most common. crease in YPLL.
Methods. Age- and sex-specific and age-adjusted crude mortality rates were calculated based on mortality and population information available from the official database of the Department of National Statistics Administration, Colombia. YPLL were estimated and adjusted for societal impact, age, and poor quality of mortality records. The results were tabulated according to codes X600-X849 and Y870 from the International Statistical Classification of Disease and Related Health Problems, 10th revision (ICD-10), and codes E950-E959 from the 9th revision (ICD-9).

Results. Suicide rates have been climbing in Colombia since 1998, particularly among young adults and males. The highest rates among males were in the age groups 20-29 years of age and over 70 years of age, and rates increased over time. Among females, the highest rates were recorded for the group 10-19 years of age. The YPLL rose in proportion with the increase in suicides, from $0.81 \%$ in 1981 to $2.20 \%$ in 2002. Among males, the most common methods used were firearms and explosives, hanging, and poison, with a relative increase in hanging; whereas among females,

Conclusions. A rising trend in suicide rates in Colombia was confirmed, especially among the productive segment of the population, which has resulted in a marked in-

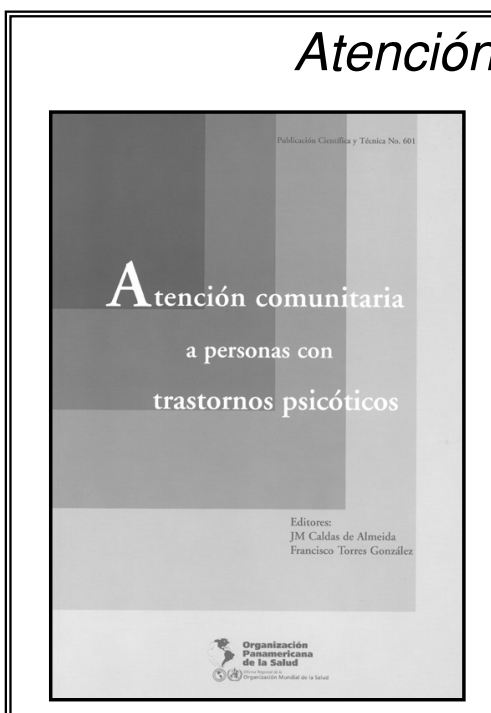

2005,152 pp.,

ISBN 9275316015

Código: PC 601

Precio: US\$18.00 en América

Latina y el Caribe/ US $\$ 25.00$ en

el resto del muncdo
Adquiera esta publicación por medio de la librería en línea de la OPS: http://publications.paho.org; correo electrónico: paho@pmds.com; Fax: (301) 209-9789; Oficina de país de la OPS/OMS 